\title{
STUdiES ON REVERSE TENSION MECHANISM OF CHEESE DYEING
}

\author{
Abu Shaid ${ }^{1}$, Md. Abu Bakar Siddiquee ${ }^{2}$, Md Azijur Rahman ${ }^{3}$ \\ ${ }^{1}$ Lecturer, Department of Textile Engineering, Dhaka University of Engineering and \\ Technology,Gazipur-1700, Gazipur, Bangladesh. ${ }^{2}$ Assistant Professor \& chair, Department of \\ Textile Engineering, Mawlana Bhashani Science \& Technology University, Tangail, Bangladesh ; \\ ${ }^{3}$ Manager, cone-dyeing, Colormaster ltd, Jamirdia, Valuka, Mymensing, Bangladesh. \\ *Corresponding Author’s e-mail: abushaidmbstu@yahoo.com
}

\begin{abstract}
In this paper reverse tension mechanism were observed. Soft winding is the main criteria of level yarn dyeing. One of the most vital difficulty of Dyeing a yarn package in cheese form is the density variation of the soft wounded packages themselves and also within a package. . In most cases this density variation results unlevelness of dyed package. This type of unlevel is very common for double ply yarn where the density varies significantly. A through investigation had made to suggest effective ways to achieve evenly dens package in soft winding section in a well equipped modern yarn dyeing factory. A new method termed as 'reverse tension mechanism' has developed to maintain even density within a package and also at packages themselves .Co-efficient of variance found near $0.3 \%$ for the new technique whereas it is more than $1 \%$ for the current system. This paper shows that the density achieved by reverse tension mechanism is very uniform at different layers of a package and also at the packages themselves than the conventional machine settings for both carded and combed cotton double ply yarn of finer and coarser count.
\end{abstract}

Keywords: Reverse tension mechanism, Portion density, Package to package \& within package. Unlevelness.

\section{Introduction}

Package to Package and within package shade variation problem is the major technical difficulty for all the yarn dyeing factories engaged in dyeing of double ply yarn packages in cheese form. The problem becomes very dangerous for those industries that are engaged in sweater manufacturing with solid color because the unlevel packages of dyed yarn make the different parts of a garment in different depth of shade ( Fig-1 a,b) and it become very visible in finer gauge structure. Traditionally it is solved in most cases by segregation of the unlevel portion from the packages or taking further reprocesses like washing, stripping or redyeing the batches. In some cases the dyers try to solve the problem by employing some extra care in dyeing process. Rejecting the unlevel portion or re-correction of levelness is definitely not a economically comfortable way to minimize the problem as it causes wastage of lot of time and money. The current research work tried to develop effective ways to minimize the density variation in soft winding package.

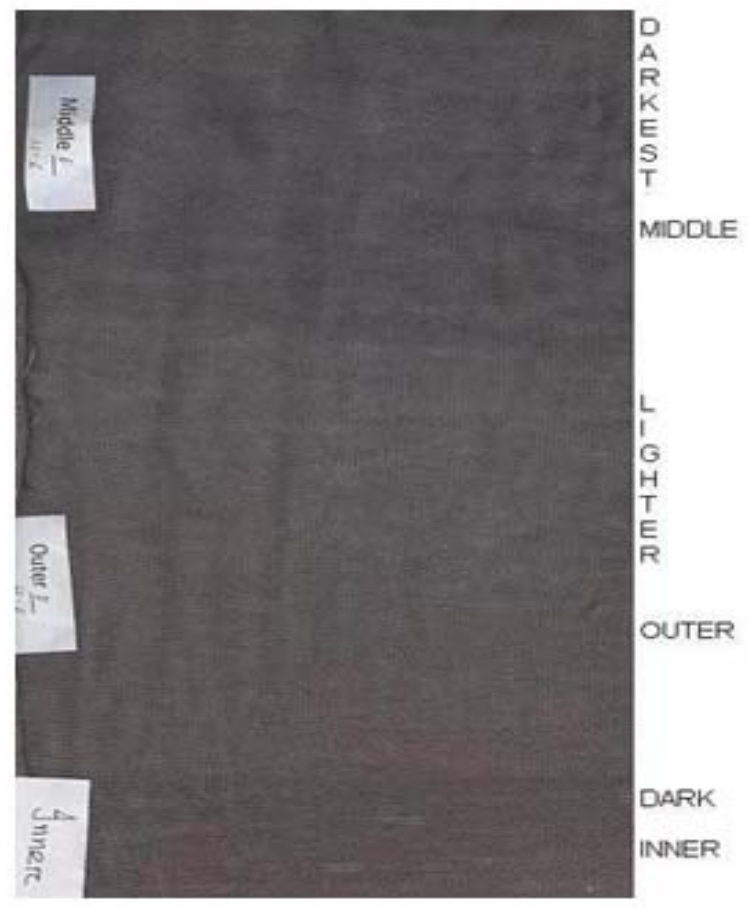

Fig:-1 (a):- Unlevelness in the knitted fabric from a unlevel dyed package 


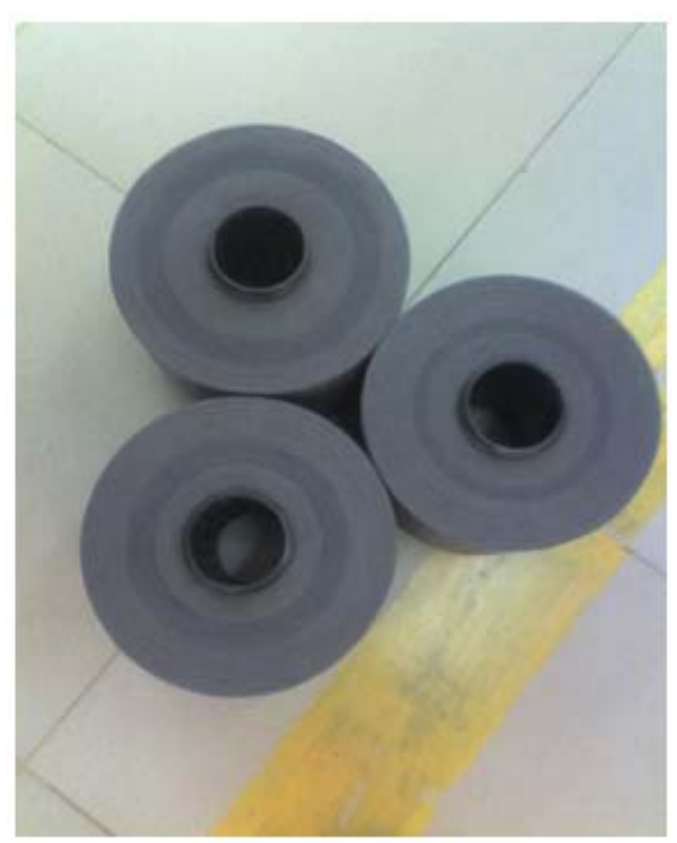

Fig: 1(b):- Unlevelness in the dyed produced packages, which lead to the unlevelness in the knitted fabric

\section{Reverse Tension Mechanism and Uniformity of Density}

Soft winding is the preparation of yarn package before dyeing. Yarns are wounded on perforated package holder with a definite weight of yarn in each package. To achieve levelness of shade after dyeing a yarn package, it is very important to made soft winding packages having uniform density at different position of the package ( portion density ) and also having minimum density variation among packages themselves package to package density). Uniformity of both portion density and package to package density is a vital factor to have levelness of shade after dyeing. In traditional soft winding machine settings, normally the initial tension is set greater than the final tension as to provide a firm base for the rest of the winding to build a stable package. But this paper shows that it is not true that the initial tension must be greater than the final tension. It has been found that a certain amount of initial tension is enough for the stable package build up and above this tension, the higher

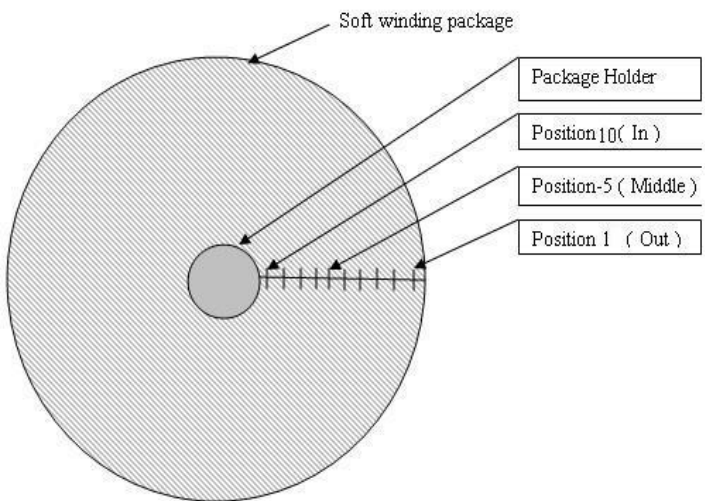

Fig:2- 'Portion density'- density at different position of a package

final tension does not hamper the stable package build up. More over, it has been found that higher final tension can provide better uniformity in portion density than the conventional setting with high initial tension. This idea of taking higher final tension is a new but very effective one. Trials have run with all the soft winding controlling parameters such as winding speed, length, winding angle, type of winding, winding tension etc. and it has been found that winding tension has maximum influence on density variation and the density variation found minimum at that cases where the final winding tension is higher than the initial tension, which is a reverse idea of the common setting. That is why it is termed as reverse tension mechanism.

\section{Importance of this Research}

This paper will be a very valuable assets the readymade garments manufacturing factories who are engaged in producing cardigans, sweaters and other knitted garments from double ply cotton yarn. The research will suggest effective techniques to achieve evenly dense pre-dyeing soft wound package. As a result, within package shade variation ,the most common and most disturbing problem of yarn dyeing sector will be minimize with a great extent.

\section{Methodology \\ "SSM PSC W/D DIGICONE Preciflex ${ }^{\text {TM }}$ precision package winder" soft winding} machine is used for preparing yarn packages. Winding tension has adjusted keeping Speed, Package diameter, Package weight as usual 
for all types of yarn. Yarn types used are $32 / 2$ and 20/2 combed and combed cotton yarn. Uneven tension causes uneven package density. which in turn results in unevenness in subsequent technological processes such as dyeing [1]. Different machine setting has been evaluated to maintain even tension as well as to achieve even density throughout the packages. The soft winding machine setting is very important for uniform package density. Again the setting vary for count of the yarn and the types of the yarn. Here different machine settings are applied for each type and count of yarn and the uniformity of the density is checked. In case of machine setting, winding tension is a vital fact for package buildup. Tension must not as high as it will resist the liquor flow and at the same time it must not too low which can make the package so soft that can causes yarn fly out from package during the flow direction alteration. This alteration is very important to ensure level dyeing as penetration is obviously better if the flow can be reversed periodically[2] Again, the yarn tension must be uniform throughout in the different layers of a soft wound package. Density in different layers of a single package and the packages in one run ( 60 packages/trial) is checked for each trial and overall more than 150 trials have made.

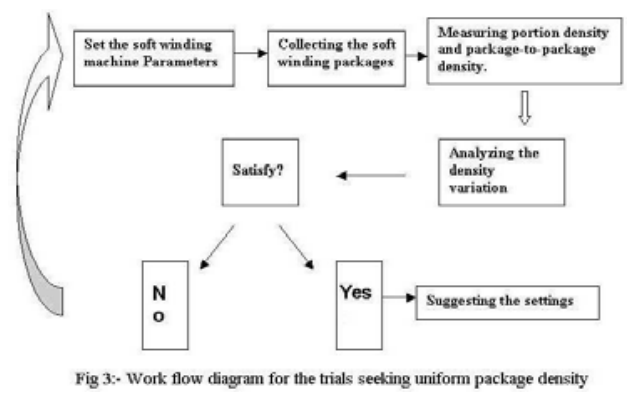

\section{Result and Discussion}

"well wound is half dyed". A uniform \& adequate flow of liquor through the wound packages of material is a necessary perquisite for level dyeing. [3]. In cheese dyeing, there is a general tendency that the yarn density and resistance to passage of a dye liquor through the yarn cheeses are lower in the inner yarn layers than in the outer yarn layers, with the result that insufficient contact of the outer yarn layers with the dye liquor occurs, whereby after dyeing, color differences are produced between the inner and outer yarn layers of each yarn cheese,[4] It is required to wind up the yarns evenly in order to bring the packages into even contact with a dye liquor. If it has not been possible to obviate unevenness in winding density, consequently, it will be extremely difficult to pursue even dyeing characteristics, since a dye liquor flows smoothly through soft portions of the cheeses, but is difficult to flow through high-density portions of the cheeses [5]. It is need to ensure uniform package density both at different layers of a package (portion density) \& among the packages themselves to avoid channeling [6]. Package density are usually range of 0.25 to $0.5 \mathrm{~kg} / \mathrm{l}$ for staple fiber [7]. But it is necessary for all the packages of a batch to be uniformly dense in packages themselves and within one package. All packages must be as identical as possible and the yarn tension throughout the package must be even [8].

The current investigation will suggest a very useful technique termed as reverse tension mechanism to ensure even density at different layers in a package and also between the packages themselves.

To check density within a package we checked at 10 to 15 portion of a soft wounded package (fig2).

The experiment had run for both finer count and coarse count :

\begin{tabular}{|l|l|}
\hline Finer count,(Ne) & Coarser count (Ne) \\
\hline $32 / 2$ (both carded & $\begin{array}{l}20 / 2 \text { (both carded \& } \\
\text { combed) }\end{array}$ \\
\hline
\end{tabular}

A typical Soft winding machine setting can be as follow:

$800-600-800$

$35 \%-25 \%$ and $80 \%$ at 70 s;

$145 \mathrm{~mm}-19000 \mathrm{~m}-180 \mathrm{~mm}$;

$50 \%$ overfeed;
$4.434-7.434$
or $16^{0}-12^{0}$
Precision winding. DIGI cone winding 
Here,

$800=$ The required production speed

$600=$ The required initial speed

$800=$ The required full package speed or final speed.

$35 \%=$ Empty tube yarn tension factor

$25 \%=$ Full package yarn tension factor

$80 \%$ = initial yarn tension factor

$70 \mathrm{~s}=$ initial yarn tension time.

$145 \mathrm{~mm}=$ The required starting traverse for determining the winding length

$19000 \mathrm{~m}=$ The required length of the yarn on the package

$180 \mathrm{~mm}=$ The required diameter results from the empty tube diameter \& the wound yarn.

Overfeed $=$ This parameter determine the difference between feeder speed and production speed. (eg: 50\% means the feeder speed is 1.5 times as large as the production speed ie overfeed, 0\%means the feeder speed and the production speed are identical and $-100 \%$ means the feeder stands still ).

$4.434=$ Winding ratio for precision winding

7.434 = initial winding ratio for precision winding $16^{0}=$ winding angle for DEGI cone winding $12^{0}=$ initial winding angle for DIGI cone winding

More than.150 trials have been run to suggest appropriate machine setting considering all the soft winding machine parameter. The results are shown in the tables \& charts below-

\subsection{Data comparison}

5.1.1. For finer count ( $32 / 2$ carded and combed cotton yarn)

Target package wt $=1170 \mathrm{gm}$

Target package density $=0.337 \mathrm{gm} / \mathrm{cc}$

The diameter of package holder is $6.8 \mathrm{~cm}$ and it is constant for all the trials.

\subsubsection{Double ply combed cotton yarn:}

Table 5.1.1.1.(a):- Portion density variation( Within package density variation):-

\begin{tabular}{|c|c|c|c|c|c|c|c|c|c|c|c|c|c|}
\hline \multicolumn{14}{|c|}{$32 / 2$ combed yarn } \\
\hline \multicolumn{7}{|c|}{ Reverse tension mechanism } & \multicolumn{7}{|c|}{ Common of Normal mechanism } \\
\hline \multirow[b]{2}{*}{$\mathrm{M} / \mathrm{C}$ setting } & \multicolumn{5}{|c|}{ Package parameter } & \multirow{2}{*}{$\begin{array}{c}\text { De } \\
\text { nsit } \\
\text { i y } \\
\text { vari } \\
\text { atio } \\
\text { n }\end{array}$} & \multirow[b]{2}{*}{ M/C setting } & \multicolumn{5}{|c|}{ Package parameter } & De \\
\hline & $\begin{array}{c}\text { Pos } \\
\text { itio } \\
\text { n }\end{array}$ & $\begin{array}{c}\text { Pkg } \\
\text { Dia } \\
\text { (cm) }\end{array}$ & $\begin{array}{c}\text { Pkg } \\
\text { height } \\
\text { (cm) }\end{array}$ & $\begin{array}{c}\mathrm{Pkg} \\
\text { wt } \\
\text { (gm) }\end{array}$ & $\begin{array}{c}\text { Densi } \\
\text { ty } \\
(\mathrm{gm} / \mathrm{c} \\
\mathrm{c})\end{array}$ & & & $\begin{array}{c}\text { Pos } \\
\text { itio } \\
\text { n }\end{array}$ & $\begin{array}{l}\text { Pak } \\
\text { Dia } \\
(\mathrm{cm})\end{array}$ & $\begin{array}{c}\text { Pkg } \\
\text { height } \\
\text { (cm) }\end{array}$ & $\begin{array}{c}p \mathrm{~kg} \\
\text { wt } \\
(\mathrm{gm})\end{array}$ & $\begin{array}{c}\text { Densi } \\
\text { ty } \\
(\mathrm{gm} / \mathrm{c} \\
\mathrm{c})\end{array}$ & $\begin{array}{c}y \\
\text { var } \\
\text { iati } \\
\text { on }\end{array}$ \\
\hline $1100-950-1050$ & \multirow{3}{*}{ Out } & 18.5 & 14.8 & 1172 & 0.338 & \multirow{9}{*}{ 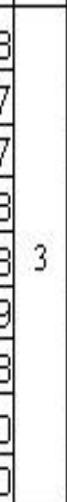 } & $1150-1000-1050$ & \multirow{3}{*}{$\begin{array}{c}\text { Out } \\
\text { er } \\
\end{array}$} & 18.5 & 15 & 1177 & 70.335 & \\
\hline \multirow{2}{*}{$\begin{array}{c}53 \%-63 \% \text { \& } \\
70 \% \text { at } 70 \mathrm{~s}\end{array}$} & & 17.8 & 14.7 & 1064 & 0.337 & & \multirow{2}{*}{$\begin{array}{c}60 \%-56 \% \& 70 \% \\
\text { at } 70 \mathrm{~s}\end{array}$} & & 17.8 & 15 & 1078 & 0.335 & \\
\hline & & 16.9 & 14.8 & 947 & 0.337 & & & & 16.8 & 15.1 & 950 & 0.336 & \\
\hline & \multirow{3}{*}{ Mi } & 15.8 & 14.9 & 814 & 0.338 & & & $\mathrm{Mi}$ & 15.7 & 15.1 & 815 & 0.339 & \\
\hline \multirow{2}{*}{$\begin{array}{c}145 \mathrm{~mm}-32400 \mathrm{~m}- \\
185 \mathrm{~mm} \\
\end{array}$} & & 14.9 & 15 & 710 & 0.338 & & \multirow{2}{*}{$\begin{array}{c}150 \mathrm{~mm}-32150 \mathrm{~m}- \\
186 \mathrm{~mm}\end{array}$} & \multirow{2}{*}{$\begin{array}{l}\text { di1 } \\
\text { dd1 }\end{array}$} & 14.8 & 15.2 & 709 & 0.339 & 9 \\
\hline & & 13.9 & 15.1 & 602 & 0.339 & & & & 13.8 & 15.3 & 600 & 0.340 & \\
\hline & \multirow{3}{*}{$\begin{array}{c}\operatorname{Inn} \\
\text { er }\end{array}$} & 12.8 & 15.1 & 482 & 0.338 & & & \multirow{3}{*}{$\begin{array}{c}\operatorname{In} n \\
\text { er }\end{array}$} & 12.9 & 15.3 & 505 & 0.342 & \\
\hline $16^{\circ}-12^{\circ}$ & & 10.2 & 15.2 & 245 & 0.340 & & $16^{\circ}-12^{\circ}$ & & 11.7 & 15.3 & 385 & 0.343 & \\
\hline $32 \%$ overfeed & & 9.3 & 15.1 & & 0.340 & & $30 \%$ overfeed & & 10.7 & 15.2 & 290 & 0.343 & \\
\hline \multicolumn{7}{|c|}{$C V \%=0.311554$} & \multicolumn{7}{|c|}{$\mathrm{CV} \%=0.894921$} \\
\hline
\end{tabular}



Table 5.1.1.1(b):- Package to package density variation

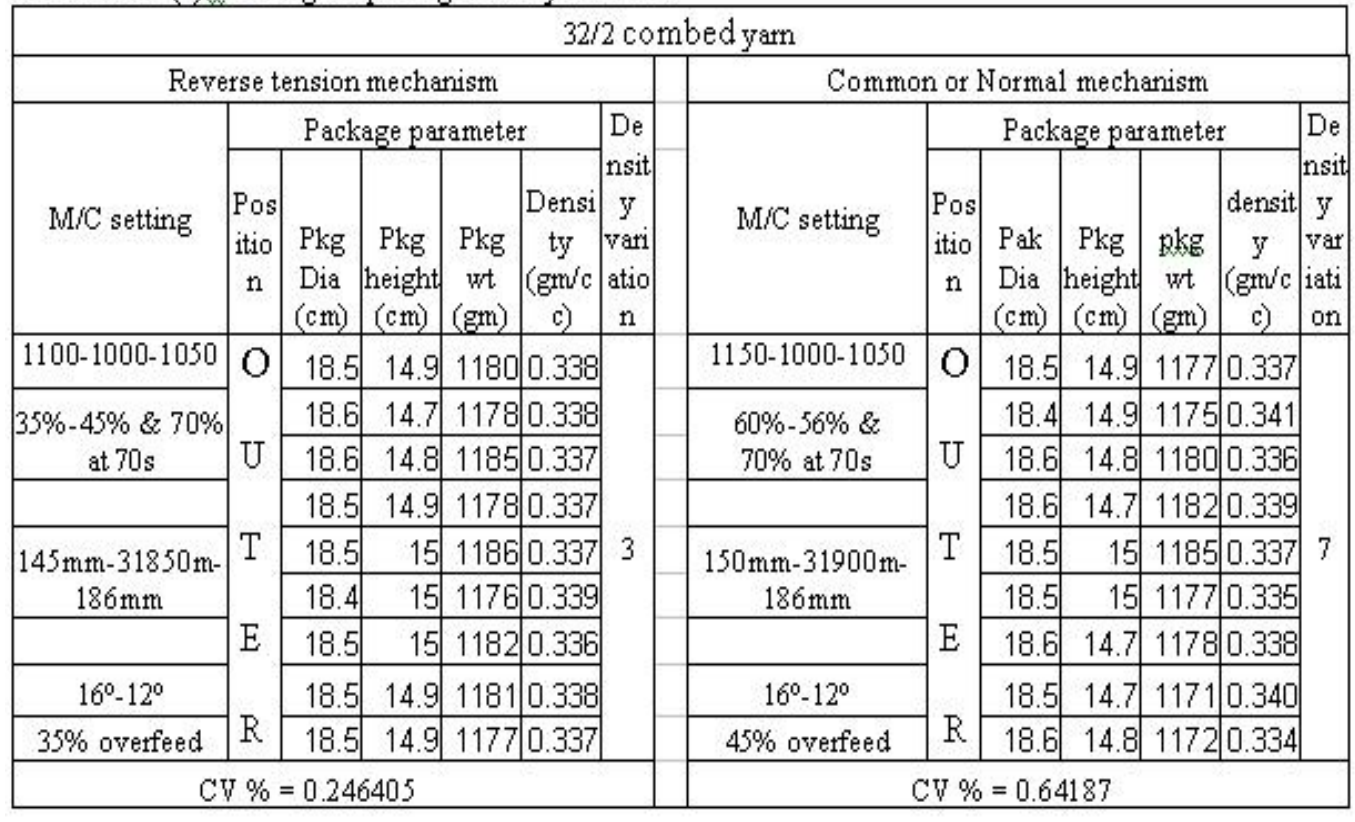

\subsubsection{Double ply carded cotton yarn:}

Table 5.1.1.2.(a):- Portion density variation (Within package density variation):-

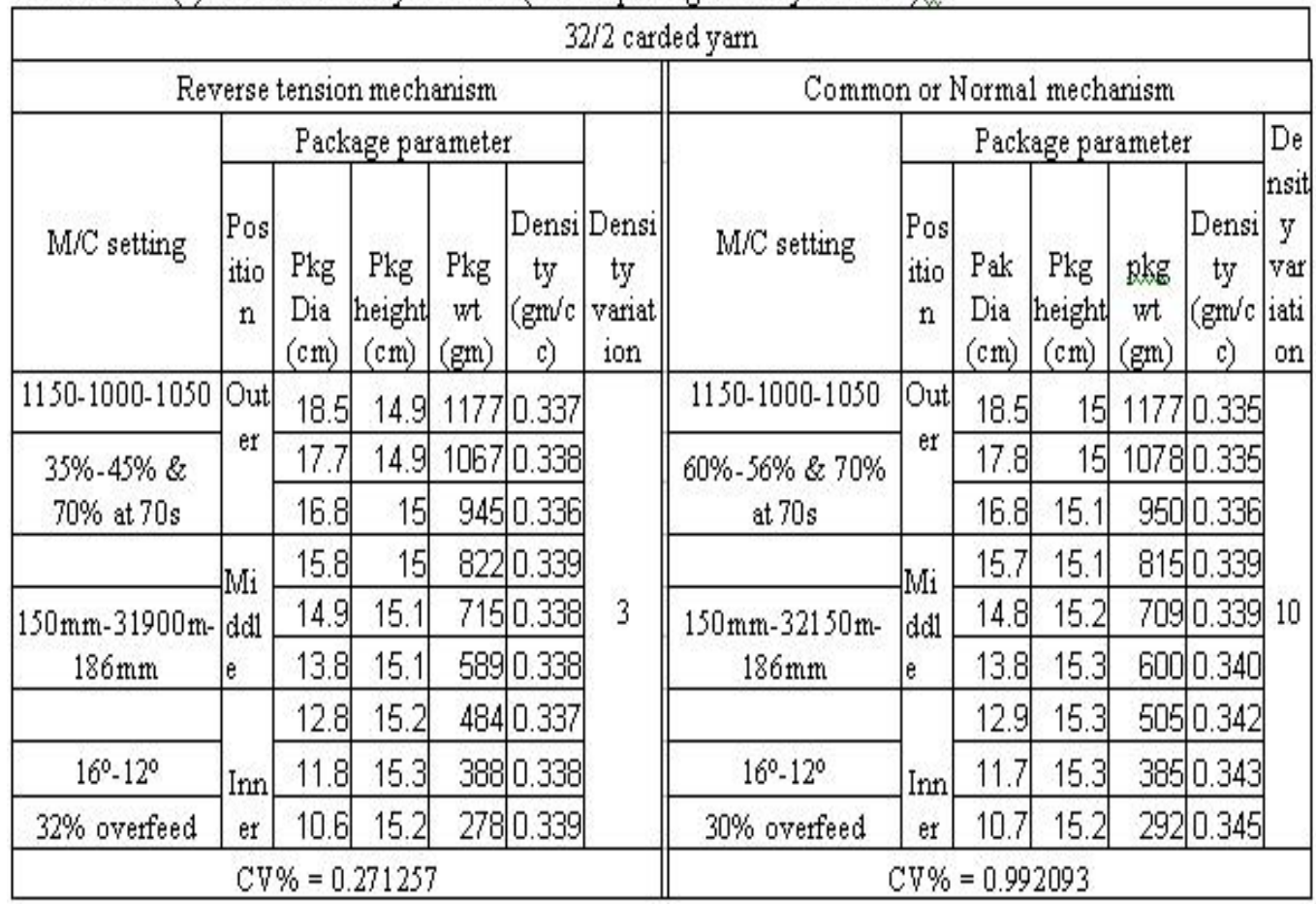


Table 5.1.1.2(b):- Package to package density variation

\begin{tabular}{|c|c|c|c|c|c|c|c|c|c|c|c|c|c|}
\hline \multicolumn{14}{|c|}{$32 / 2$ carded yam } \\
\hline \multicolumn{7}{|c|}{ Reverse tension mechanism } & \multicolumn{7}{|c|}{ Common or Normal mechanism } \\
\hline \multirow[b]{2}{*}{ M/C setting } & \multicolumn{5}{|c|}{ Package parameter } & \multirow[b]{2}{*}{$\mid \begin{array}{c}\text { Densi } \\
\text { ty } \\
\text { variat } \\
\text { ion }\end{array}$} & \multirow[b]{2}{*}{ M/C setting } & \multicolumn{5}{|c|}{ Package parameter } & De \\
\hline & $\begin{array}{c}\text { Pos } \\
\text { itio } \\
\text { n }\end{array}$ & $\begin{array}{l}\text { Pkg } \\
\text { Dia } \\
\text { (cm) }\end{array}$ & \begin{tabular}{|c|} 
Pkg \\
height \\
(cm)
\end{tabular} & $\begin{array}{l}\text { Pkg } \\
w t \\
(g m)\end{array}$ & $\begin{array}{c}\text { Densi } \\
\text { ty } \\
(\mathrm{gm} / \mathrm{c} \\
\mathrm{c})\end{array}$ & & & $\begin{array}{c}\text { Pos } \\
\text { itio } \\
\text { n }\end{array}$ & $\begin{array}{c}\text { Pak } \\
\text { Dia } \\
(\mathrm{cm})\end{array}$ & $\begin{array}{c}\text { Pkg } \\
\text { height } \\
\text { (cm) }\end{array}$ & $\begin{array}{l}\mathrm{pkg} \\
\mathrm{wt} \\
(\mathrm{gm})\end{array}$ & $\mid \begin{array}{c}\text { Densi } \\
\text { ty } \\
(\mathrm{gtr} / \mathrm{c} \\
\mathrm{c})\end{array}$ & $\begin{array}{c}y \\
\text { var } \\
\text { iati } \\
\text { on } \\
\end{array}$ \\
\hline $1100-850-950$ & \multirow[t]{4}{*}{$\mathrm{O}$} & 18.5 & 14.8 & 1174 & 0.338 & & $1150-1000-1050$ & \multirow[t]{2}{*}{$\mathrm{O}$} & 18.5 & 14.9 & 1177 & 0.337 & \\
\hline \multirow{3}{*}{$\begin{array}{c}62 \%-70 \% \& 90 \% \\
\text { at } 70 \mathrm{~s}\end{array}$} & & 18.6 & 14.7 & 1175 & 0.337 & & $60 \%-2$ & & 18.7 & 14.6 & 1175 & 0.335 & \\
\hline & & 18.6 & 14.8 & 1179 & 0.336 & & at $70 \mathrm{~s}$ & \multirow[t]{2}{*}{$\mathrm{U}$} & & 14.8 & 1180 & 0.332 & \\
\hline & & 18.6 & 14.8 & 1180 & 0.336 & & & & 18.6 & 14.9 & 1186 & 0.335 & \\
\hline \multirow{3}{*}{$\begin{array}{c}145 \mathrm{~mm}-31800 \mathrm{~m}- \\
187 \mathrm{~mm}\end{array}$} & \multirow[t]{2}{*}{$\mathrm{T}$} & 18.7 & 14.6 & 1174 & 0.335 & 4 & $150 \mathrm{~mm}-32150 \mathrm{~m}-$ & \multirow[t]{2}{*}{$\mathrm{T}$} & 18.5 & 14.6 & 1168 & 0.341 & 9 \\
\hline & & 18.5 & 15 & 1187 & 0.337 & & $186 \mathrm{~mm}$ & & 18.6 & 14.8 & 1171 & 0.333 & \\
\hline & \multirow[b]{3}{*}{$\mathrm{R}$} & 18.6 & 14.7 & 1172 & 0.336 & & & \multirow[b]{3}{*}{$\mathrm{R}$} & 18.6 & 14.6 & 1170 & 0.338 & \\
\hline $16^{\circ}-12^{\circ}$ & & 18.6 & 14.6 & 1175 & 0.339 & & $16^{\circ}-12^{\circ}$ & & 18.5 & 14.8 & 1182 & 0.341 & \\
\hline $32 \%$ overfeed & & 18.7 & 14.6 & 1178 & 0.336 & & $30 \%$ overfeed & & 18.7 & 14.6 & 1170 & 0.333 & \\
\hline \multicolumn{7}{|c|}{$\mathrm{CV} \%=0.34298$} & \multicolumn{7}{|c|}{$\mathrm{CV} \%=0.945478$} \\
\hline
\end{tabular}

5.1.2. For coarser count (20/2 carded and combed cotton yarn)

Target package $\mathrm{wt}=1100 \mathrm{gm} \quad$ Target package density $=0.337 \mathrm{gm} / \mathrm{cc}$

The diameter of package holder is $6.8 \mathrm{~cm}$ and it is constant for all the trials.

\subsubsection{1 :Double ply combed cotton yarn}

Table 5.1.2.1.(a):- Portion density variation (Within package density variation):-

\begin{tabular}{|c|c|c|c|c|c|c|c|c|c|c|c|c|c|}
\hline \multicolumn{14}{|c|}{$20 / 2$ combed yarn } \\
\hline \multicolumn{7}{|c|}{ Reverse tension mechanism } & \multicolumn{7}{|c|}{ Common or Normal mechanism } \\
\hline \multirow[b]{2}{*}{ M/C setting } & \multicolumn{5}{|c|}{ Package parameter } & \multirow{2}{*}{$\begin{array}{c}\text { De } \\
\text { nsit } \\
\text { y } \\
\text { vari } \\
\text { atio } \\
\text { n }\end{array}$} & \multirow[b]{2}{*}{ M/C setting } & \multicolumn{5}{|c|}{ Package parameter } & $D$ \\
\hline & $\begin{array}{c}\text { Pos } \\
\text { itio } \\
\text { n }\end{array}$ & $\begin{array}{l}\text { Pkg } \\
\text { Dia } \\
\text { (cm) }\end{array}$ & $\begin{array}{c}\text { Pkg } \\
\text { height } \\
\text { (cm) }\end{array}$ & $\begin{array}{c}\text { Pkg } \\
\text { wh } \\
(\mathrm{gm})\end{array}$ & $\begin{array}{c}\text { Densi } \\
\text { ty } \\
(\mathrm{gm} / \mathrm{c} \\
\mathrm{c})\end{array}$ & & & $\left|\begin{array}{c}\text { Pos } \\
\text { itio } \\
\text { n }\end{array}\right|$ & $\begin{array}{l}\text { Pak } \\
\text { Dia } \\
(\mathrm{cm})\end{array}$ & $\begin{array}{c}\text { Pkg } \\
\text { height } \\
\text { (cm) }\end{array}$ & $\begin{array}{c}\text { plog } \\
\text { wt } \\
\text { (gm) }\end{array}$ & $\mid \begin{array}{c}\text { Densi } \\
\text { ty } \\
(\mathrm{gm} / \mathrm{c} \\
\mathrm{c})\end{array}$ & $\begin{array}{c}y \\
\text { var } \\
\text { iati } \\
\text { on }\end{array}$ \\
\hline $1100-900-1050$ & \multirow{3}{*}{ Out } & 18.1 & 14.7 & 1102 & 0.336 & \multirow{9}{*}{ 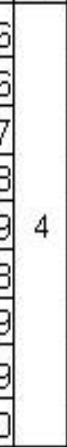 } & $1100-950-1050$ & \multirow{3}{*}{$\begin{array}{l}\text { Out } \\
\text { er }\end{array}$} & 18.5 & 14.9 & 117 & 0.337 & \\
\hline \multirow{2}{*}{$\begin{array}{c}53 \%-49 \% \text { \& } \\
80 \% \text { at } 70 \mathrm{~s}\end{array}$} & & 17.4 & 14.7 & 1005 & 0.336 & & \multirow{2}{*}{$\begin{array}{l}48 \%-58 \% \& \& \\
80 \% \text { at } 70 \mathrm{~s}\end{array}$} & & 17.8 & 14.9 & 1068 & 0.334 & \\
\hline & & 16.6 & 14.8 & 908 & 0.337 & & & & 16.8 & 15 & 94 & 0.336 & \\
\hline & \multirow{3}{*}{$\begin{array}{l}\mathrm{Mi} \\
\mathrm{dd} \mathrm{d} \\
\mathrm{e}\end{array}$} & 15.6 & 14.8 & 785 & 0.338 & & & \multirow{3}{*}{$\begin{array}{l}\mathrm{Mi} \\
\mathrm{d} d \mathrm{~d} \\
\mathrm{e}\end{array}$} & 15.9 & 15 & 82 & 0.335 & \\
\hline \multirow{2}{*}{$\begin{array}{c}145 \mathrm{~mm}-18100 \mathrm{~m}- \\
181 \mathrm{~mm}\end{array}$} & & 14.8 & 14.9 & 695 & 0.339 & & \multirow{2}{*}{$9600 \mathrm{~m}-$} & & 14.9 & 15.1 & 717 & 0.339 & 9 \\
\hline & & 13.8 & 15 & 585 & 0.338 & & & & 13.8 & 15.1 & 59 & 20.340 & \\
\hline & \multirow{3}{*}{$\begin{array}{l}\text { Inn } \\
\text { er }\end{array}$} & 12.9 & 15 & 490 & 0.339 & & & \multirow{3}{*}{$\begin{array}{c}\text { Inn } \\
\text { er }\end{array}$} & 12.8 & 15.2 & 490 & 0.341 & \\
\hline $16^{\circ}-12^{\circ}$ & & 11.3 & 15.1 & 338 & 0.339 & & $16^{\circ}-12^{\circ}$ & & 11.8 & 15.3 & 39. & 40.343 & \\
\hline $35 \%$ overfeed & & 10.2 & 15.1 & 244 & 0.340 & & $35 \%$ overfeed & & 10.5 & 15.2 & 273 & 3.343 & \\
\hline \multicolumn{7}{|c|}{$\mathrm{CV} \%=0.394477$} & \multicolumn{7}{|c|}{$\mathrm{CV} \%=0.933743$} \\
\hline
\end{tabular}


Table 5.1.2.1(b):- Package to package density variation

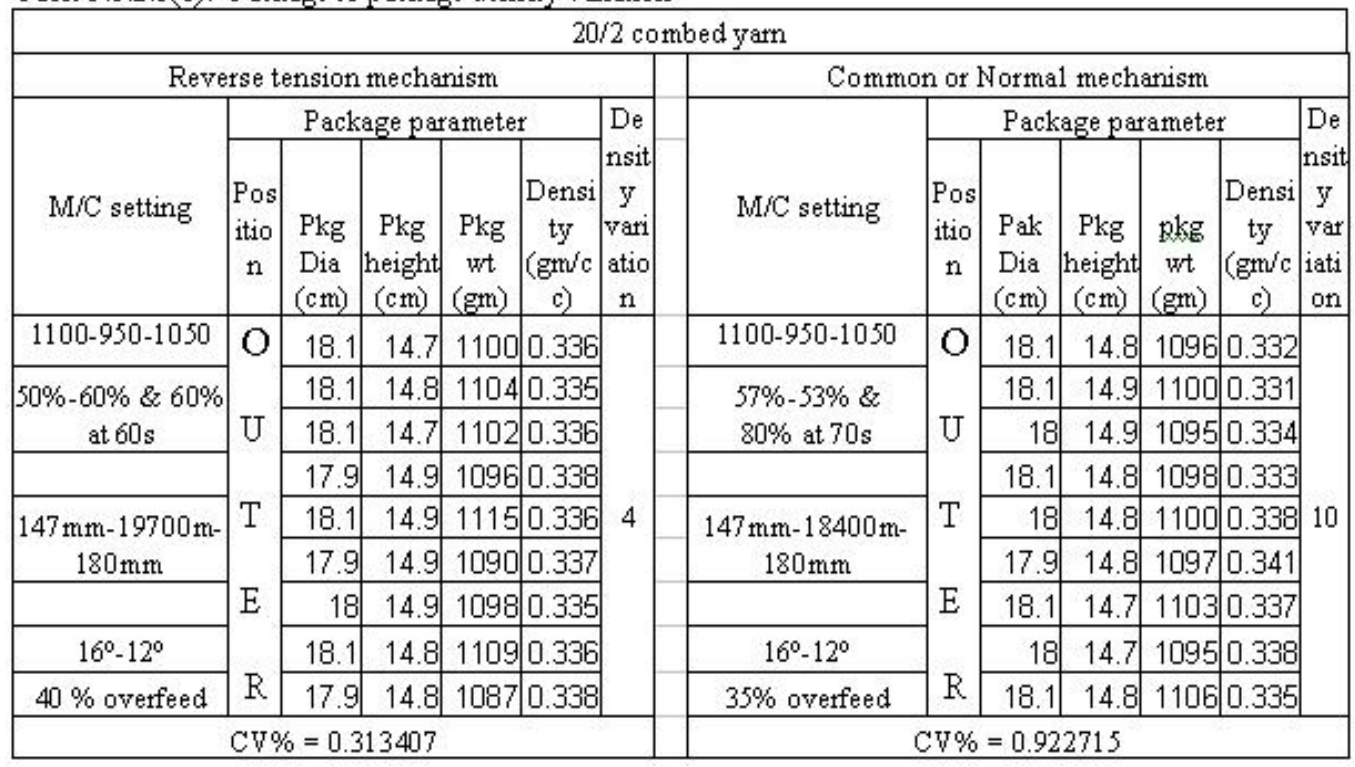

\subsubsection{Double ply carded cotton yarn:}

Table 5.1.2.2.(a):- Portion density variation (Within package density variation) :-

\begin{tabular}{|c|c|c|c|c|c|c|c|c|c|c|c|c|c|}
\hline \multicolumn{14}{|c|}{$20 / 2$ carded yam } \\
\hline \multicolumn{7}{|c|}{ Reverse tension mechanism } & \multicolumn{7}{|c|}{ Common or Normal mechanism } \\
\hline \multirow[b]{2}{*}{ M/C setting } & \multicolumn{5}{|c|}{ Package parameter } & \multirow[b]{2}{*}{$\begin{array}{c}\text { Densi } \\
\text { ty } \\
\text { variat } \\
\text { ion } \\
\end{array}$} & \multirow[b]{2}{*}{ M/C setting } & \multicolumn{5}{|c|}{ Package parameter } & \multirow{2}{*}{\begin{tabular}{|c|}
$\mathrm{De}$ \\
nsit \\
$\mathrm{y}$ \\
var \\
iati \\
on \\
\end{tabular}} \\
\hline & $\begin{array}{c}\text { Pos } \\
\text { itio } \\
\mathrm{n}\end{array}$ & $\begin{array}{l}\text { Pkg } \\
\text { Dia } \\
(\mathrm{cm}) \\
\end{array}$ & $\begin{array}{c}\text { Pkg } \\
\text { height } \\
\text { (cm) }\end{array}$ & $\begin{array}{c}\text { Pkg } \\
w t \\
(\mathrm{gm})\end{array}$ & $\begin{array}{c}\text { Densi } \\
\text { ty } \\
(\mathrm{gm} / \mathrm{c} \\
\mathrm{c})\end{array}$ & & & $\mid \begin{array}{c}\text { Pos } \\
\text { itio } \\
n\end{array}$ & $\begin{array}{c}\text { Pak } \\
\text { Dia } \\
(\mathrm{cm})\end{array}$ & $\begin{array}{c}\text { Pkg } \\
\text { height } \\
\text { (cm) }\end{array}$ & $\begin{array}{c}\mathrm{pkg} \\
\mathrm{wt} \\
(\mathrm{gm}) \\
\end{array}$ & $\begin{array}{c}\text { Densi } \\
\text { ty } \\
(\mathrm{gm} / \mathrm{c} \\
\mathrm{c})\end{array}$ & \\
\hline $1100-800-900$ & \multirow{3}{*}{$\begin{array}{c}\text { Out } \\
\text { er }\end{array}$} & 18 & 14.8 & 1097 & 0.337 & \multirow{9}{*}{ 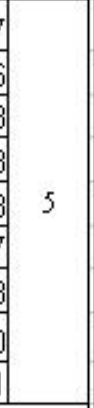 } & $1100-950-1050$ & \multirow{3}{*}{$\begin{array}{c}\text { Out } \\
\text { er }\end{array}$} & 18 & 14.8 & 1090 & 0.335 & \\
\hline \multirow{2}{*}{$\begin{array}{l}47 \%-57 \% \text { \& } \\
50 \% \text { at } 60 \mathrm{~s}\end{array}$} & & 17.5 & 14.8 & 1024 & 0.336 & & \multirow{2}{*}{$\begin{array}{l}54 \%-50 \% \text { \& } \\
80 \% \text { at } 70 s\end{array}$} & & 17.2 & 14.8 & 978 & 0.334 & \\
\hline & & 16.5 & 14.9 & 905 & 0.338 & & & & 16.5 & 14.8 & 895 & 0.337 & \\
\hline & \multirow{3}{*}{$\begin{array}{l}\mathrm{Mi} \\
\mathrm{dd} 1 \\
\mathrm{e}\end{array}$} & 15.4 & 14.9 & 766 & 0.338 & & & $\mathrm{Mi}$ & 15.4 & 15 & 773 & 0.339 & \\
\hline \multirow{2}{*}{$\begin{array}{c}145 \mathrm{~mm}-18600 \mathrm{~m}- \\
182 \mathrm{~mm}\end{array}$} & & 14.7 & 15 & 686 & 0.338 & & \multirow{2}{*}{$\begin{array}{c}145 \mathrm{~mm}-18950 \mathrm{~m}- \\
182 \mathrm{~mm}\end{array}$} & \multirow{2}{*}{$e^{d d 1}$} & 14.7 & 15 & 688 & 0.339 & \multirow{2}{*}{3} \\
\hline & & 13.7 & 15 & 572 & 0.337 & & & & 13.8 & 14.9 & 590 & 0.343 & \\
\hline & \multirow{3}{*}{$\begin{array}{c}\text { Inn } \\
\text { er }\end{array}$} & 10.2 & 15.1 & 242 & 0.338 & & & \multirow{3}{*}{$\begin{array}{c}\text { Inn } \\
\text { er }\end{array}$} & 12.6 & 15 & 465 & 0.343 & \\
\hline $16^{\circ}-12^{\circ}$ & & 9.3 & 15 & 172 & 0.340 & & $16^{\circ}-12^{\circ}$ & & 11.3 & 15 & 340 & 0.343 & \\
\hline $32 \%$ overfeed & & 8.4 & 15.1 & 109 & 0.341 & & $32 \%$ overfeed & & 9.5 & 14.9 & 188 & 0.344 & \\
\hline \multicolumn{7}{|c|}{$\mathrm{CV} \%=0.428472$} & \multicolumn{7}{|c|}{$\mathrm{CV} \%=1.0478$} \\
\hline
\end{tabular}


Table 5.1.2.2(b):- Package to package density variation

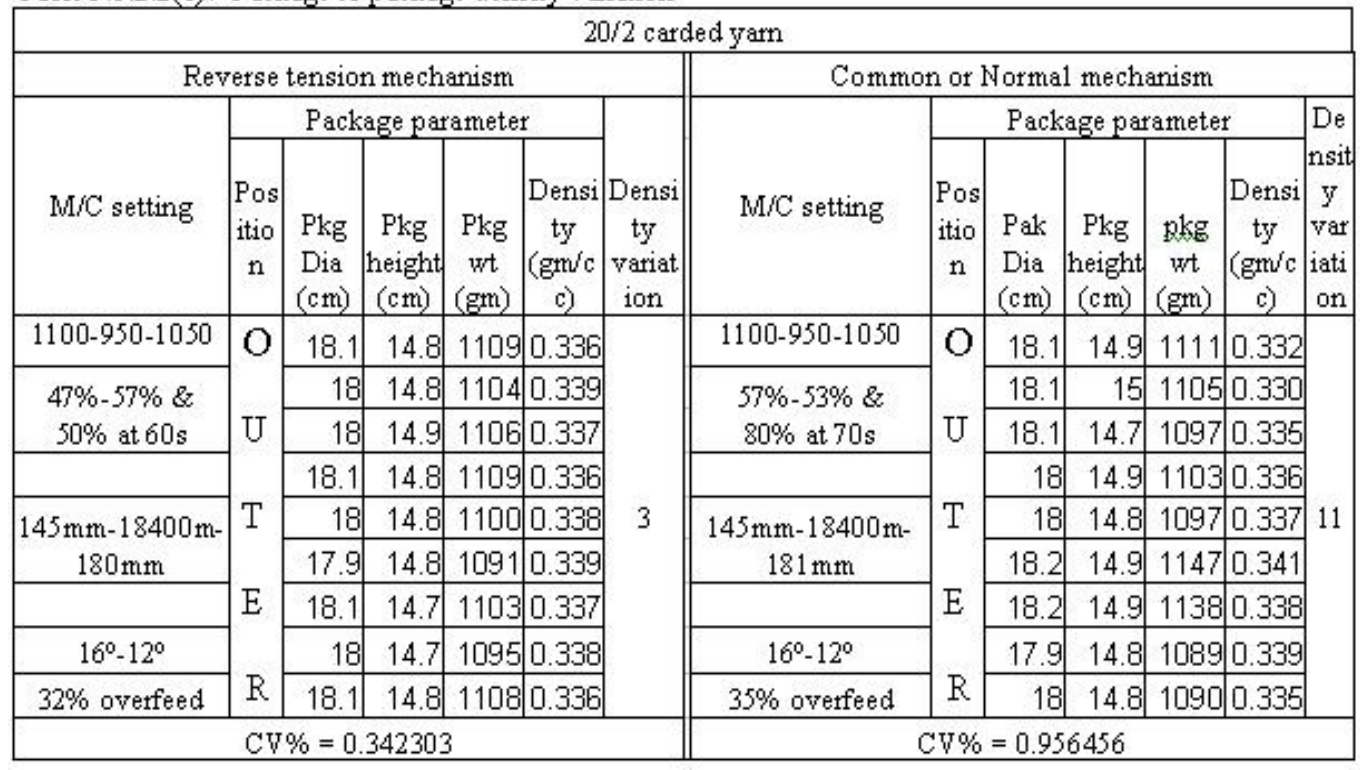

\subsection{Graphical comparison}
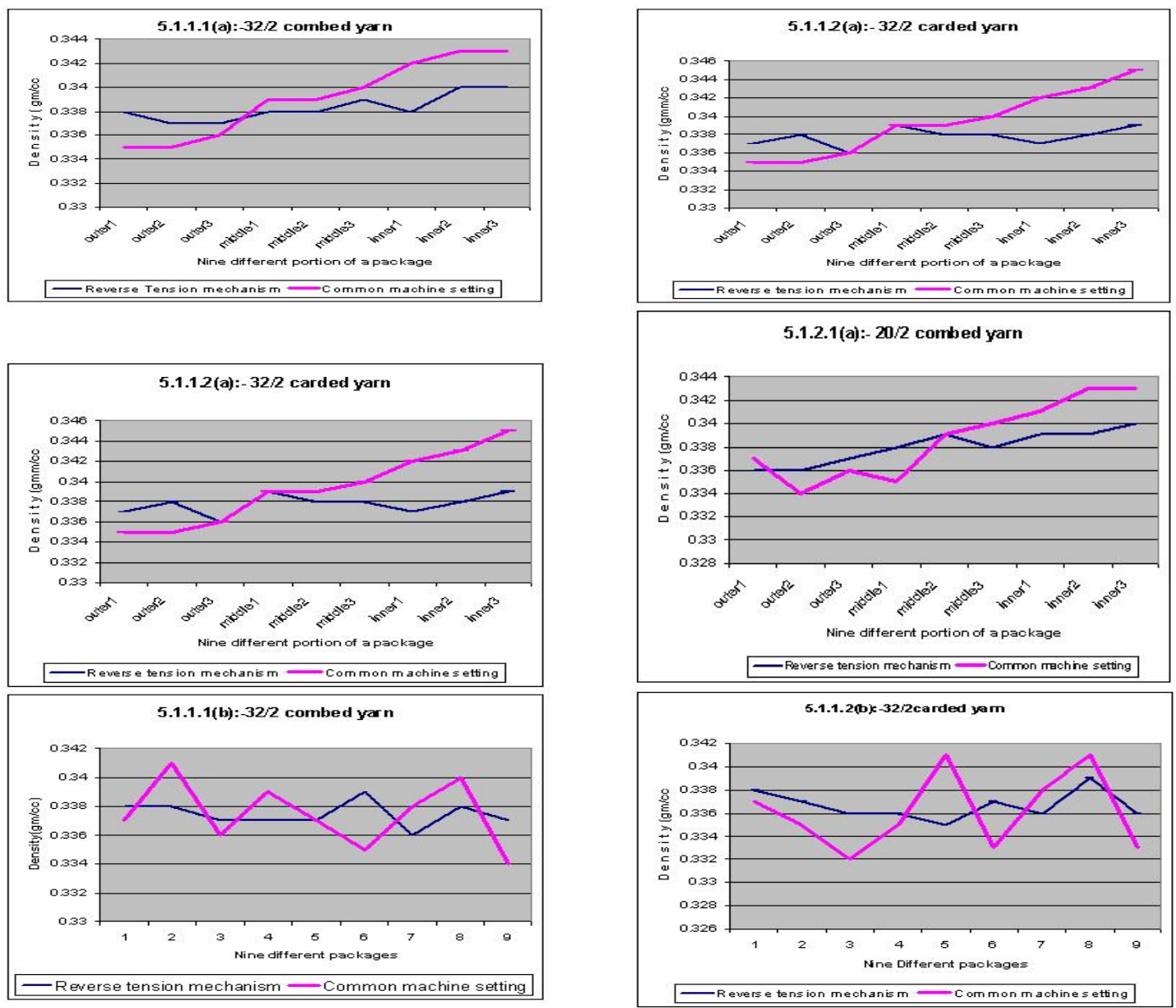


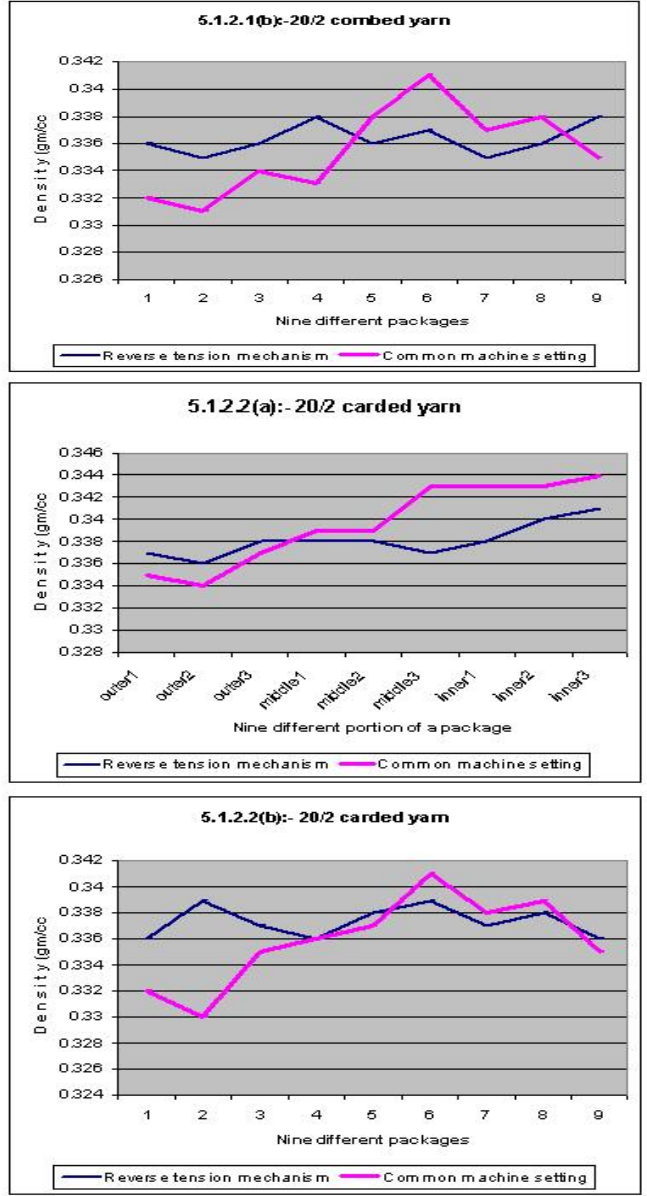

\section{3 Data Analysis}

From the above data, analytical charts are build to compare coefficient of variance for different count and types of yarn. The graphs are given below-

\subsubsection{Discussion on Tables and Graphs}

The tables and graphs given above shows that the conventional machine setting causes more density variation than the controlled machine setting (Reverse tension machine settings). The density variation within a package varies uniformly within a short limit $0.335 \mathrm{gm} / \mathrm{cc}$ to $0.340 \mathrm{gm} / \mathrm{cc}$ ) around target density of 0.337 $\mathrm{gm} / \mathrm{cc}$. But this type of variation causes very non-uniformly and with a greater range (0.334 $\mathrm{gm} / \mathrm{cc}$ to $0.345 \mathrm{gm} / \mathrm{cc}$ ) in case of common machine setting for soft winding. This type of

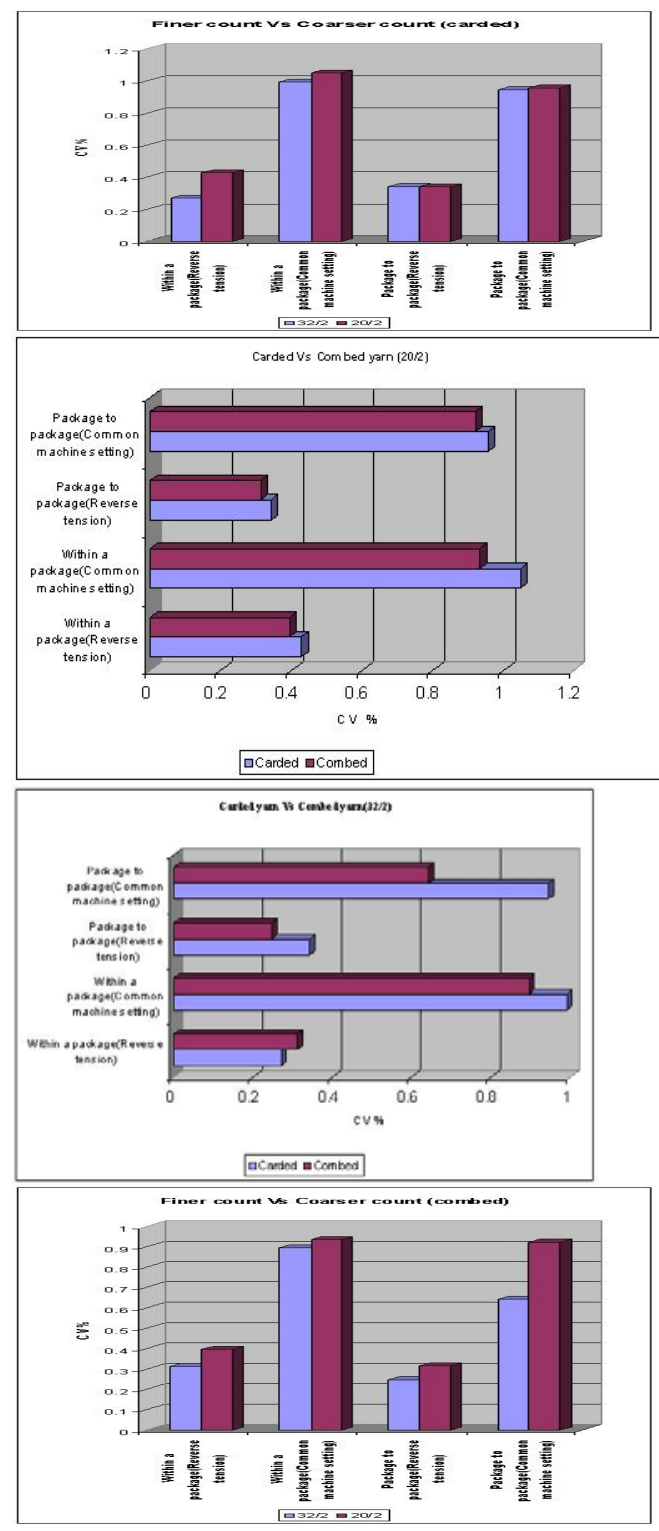

non-uniform density variation causes nonuniform velocity of the dye liquor during dyeing and thus leads to the shade variation in different layers in a single package i.e, leads to the unlevel dyeing. The machine setting suggested by the Reverse tension mechanism can ensure even density in different portion in a single yarn package and thus can ensure uniform liquor flow and provides satisfactory levelness in cone/cheese dyeing. Again the Reverse tension mechanism also ensure minimum density variation among the soft 
wounded packages themselves ( 0.335 to 0.339 $\mathrm{gm} / \mathrm{cc}$, target $0.337 \mathrm{gm} / \mathrm{cc}$ ) while the common machine setting of soft winding machine provides a larger range of density variation (0.330 to $0.341 \mathrm{gm} / \mathrm{cc}$, target $0.337 \mathrm{gm} / \mathrm{cc}$ ). This type of density variation among the soft wounded packages themselves leads to the 'cone to cone shade variation' problem.

Again, the rate of flow of liquor per unit are depends of several points. Any variation to these points may lead to the unlevel. In the ideal system, it can be shown [9] that,

$$
\mathrm{F}=(\mathrm{KPN} \pi \mathrm{D}) /(\mathrm{VT})
$$

Where, $\mathrm{F}=$ rate of flow of liquor per unit area

$\mathrm{K}=$ Constant

$\mathrm{P}=$ Pressure difference between inside

\& outside of the package

$\mathrm{N}=$ No. of Channels per unit cross-

section,

$\mathrm{D}=$ Diameter of Channels per unit

cross-section

$\mathrm{V}=$ Viscosity of liquor

$\mathrm{T}=$ Wall thickness of the package.

If the wall thickness of the packages vary (package to package density variation) or the rate of flow of liquor/unit area varies within a package (portion density variation), they certainly will causes unlevel both in packages themselves and indifferent layers in a package.

The Graph of different count of cotton yarn and with different types (carded and combed) also shows how the density varies at considerably higher rate for normal machine setting in comparison to the suggested soft winding machine setting of Reverse tension mechanism.

Again by comparing the coefficient of variance for different categories of density variation for both carded and combed yarn, it is also found that the coefficient of variation is higher in case of common or normal soft winding machine setting than the machine setting suggested in Reverse tension mechanism. It is also found that the combed yarn shows less variation than carded yarn and the finer yarn count provides more uniform winding.

\subsubsection{Outcomes}

$\checkmark$ More uniform density at different layers of a single soft wounded pre dyeing yarn package (portion density) can be build by the soft winding machine settings suggested in Reverse Tension Mechanism than conventional machine settings.

$\checkmark$ Reverse Tension Mechanism can also ensure satisfactorily similar density soft wounded packages(package to package density) in a batch.

$\checkmark$ As Reverse Tension Mechanism can provides uniformly dense packages, it minimize the risk of unlevel package dyeing at both package to package and at different layers in a single package.

$\checkmark$ Package to package and within a package density (portion density) variation is higher in a package from coarser count than a similar package from finer count.

$\checkmark$ Package to package and within a package density (portion density) variation is higher in a package from carded yarn than a similar count package from combed yarn.

\section{Concluding Remarks}

Level dyeing is the key to success for a dye house. Reverse tension mechanism can ensure uniform density which is the basic rock for the foundation of level dyeing.

\section{Acknowledgement:}

This research project was wholly launched by the "COLORMASTER LTD", a sister concern of SQ group. Financial helps for technical supports from few German and British experts are also sponsored by the group. Local 
technical helping advisors were from "Dhaka University of Engineering \& Technology”.

\section{References}

[1] Babaarslan, Osman , 1998.“Tension Variations During Cone Winding on an Open-End Spinning System” Textile Research Journal, Vol. 68, No. 9, 649-654.

[2] Trotman, E.R.” Dyeing \& Chemical Technology of Textile fibre"

[3] "Process for the uniform dyeing of textile wound packages or packaged material” United states patent 4135268,

http://www.wikipatents.com/4135268.html
[4] "Method for evenly dyeing yarn cheeses”, United states patent 5784735 ,

http://www.freepatentsonline.com/5784735.html

[5] "Methods of dyeing yarn cheeses", United states patent 5351351,

http://www.freepatentsonline.com/6921421.html

[6] Pavan S. Chinta and Sapna Dhar, DKTE's Textile and Engineering institute, Ichalkaranji.

[7] http://www.fibre2fashion.com/industryarticle/pdfd ownload.asp?filename $=1678 \&$ article $=1678$

[8] Choudhury, A. K. Roy, 2006, Textile Preparation and Dyeing, (New Delhi)

[9] Broadbent. Arthur D. "Basic Principles of Textile Coloration"

[10] Duckworth C.. (Ed.) 1983, Engineering in Textile Coloration (Bradford, UK, SDC 